\title{
La suplementación materna con ácido docosahexaenoi- co (DHA) carece de beneficio clínicamente significativo
}

\author{
Marcelo E. Farías PhD, José A. Poblete, Jorge A. Carvajal PhD. \\ Unidad de Medicina Materno-Fetal, División de Obstetricia y Ginecología, Facultad de Medicina, Pontificia Universidad \\ Católica de Chile.
}

\section{RESUMEN}

Objetivo: Evaluar los beneficios maternos y fetales de la suplementación prenatal con ácido docosahexaenoico (DHA). Método: Revisión sistemática de investigaciones clínicas controladas aleatorizadas. Resultados: La suplementación prenatal con DHA incrementó los niveles de DHA en sangre materna, en la leche materna o células neonatales. La suplementación con DHA no redujo los síntomas depresivos maternos ni mejoró el desempeño neurológico y visual de los niños. Aunque se apreció un menor riesgo de retraso cognitivo entre los hijos de mujeres suplementadas con DHA (RR 0,4; IC 95\% 0,22-0,78) y un mejor desempeño en el procesamiento mental a los 4 años, el seguimiento a 7 años mostró ausencia de diferencias significativas en el nivel intelectual. El análisis secundario de dos estudios mostró que la suplementación con DHA redujo el riesgo de parto prematuro < 34 semanas (RR 0,49; IC95\% 0,25-0,94; $p=0,03$ ), ingreso a UCI neonatal (RR 0,57; IC95\% 0,34-0,97; $p=0,04)$, peso < 2500 g (RR 0,65; IC95\% 0,44-0,96; $p=0,03$ ) y restricción de crecimiento intrauterino en pacientes primigestas (RR 0,5; IC 95\% 0,3-1,0; $p=0,03$ ). Sin embargo, la prevención de parto prematuro no fue reproducida en estudio diseñado específicamente para ello. Conclusiones: Los estudios reportan un mayor contenido de DHA materno y neonatal en respuesta a la suplementación prenatal con este ácido graso. Sin embargo, la ausencia actual de efectos clínicos relevantes no permite apoyar ni descartar completamente esta intervención durante el embarazo.

\section{PALABRAS CLAVES: Suplementación prenatal, DHA, omega-3, resultado neonatal}

\section{SUMMARY}

Aims: To evaluate maternal and fetal benefits of prenatal supplementation with docosahexaenoic acid (DHA). Method: Systematic review of clinical randomized controlled trials. Results: Prenatal DHA supplementation increased DHA levels on maternal blood, breast milk or neonatal cells. Maternal supplementation with DHA neither reduced mother's depressive symptoms nor improved the neurological and visual performance of the children. Although it was observed a reduction in risk of cognitive delay between infants of women supplemented with DHA (RR 0.4; IC95 \% 0.22-0.78) and a better performance in the mental processing at the age of 4 , the 7 years follow-up showed absence of significant differences in the intellectual level. The secondary analysis of two studies showed that the supplementation with DHA reduced the risk of premature birth <34 weeks (RR 0.49; IC95\% 0.25-0.94; $p=0.03$ ), neonatal ICU hospitalizations (RR 0.57; IC95\% 0.34-0.97; $p=0.04)$, birth weight <2500 g (RR 0.65; IC95\% 0.44-0.96; $p=0.03$ ) and intrauterine growth restriction in nulliparous patients (RR 0.5; IC95\% 0.3-1.0; $p=0.03$ ). Nevertheless, prevention of premature birth was not reproduced in a specifically designed study. Conclusions: Studies report an increased mother 
and neonatal content of DHA in response to prenatal supplementation with this polyunsaturated long chain fatty acid. Nevertheless, at date the absence of relevant clinical effects do not permit to support or to reject maternal dietary supplementation with DHA during pregnancy.

\section{KEY WORDS: Prenatal supplementation, omega-3, DHA, new born outcome}

\section{INTRODUCCIÓN}

El ácido docosahexaenoico (DHA, docosahexaenoic acid) es un ácido graso esencial de la familia de ácidos grasos poli-insaturados de cadena larga (LC-PUFAs, long chain-polyunsaturated fatty acids) o ácidos grasos omega-3 (1). A esta familia también pertenece el ácido eicosapentaenoico (EPA, eicosapentaenoic acid) y el ácido alfa-linoleico (a-LA, alpha-linoleic acid). Estos ácidos grasos son considerados esenciales debido a que nuestro organismo es incapaz de sintetizarlos; por esto, los LC-PUFAs deben ser adquiridos a través de la dieta, siendo sus principales fuentes algunos peces y algunos productos vegetales como nueces, semillas de linaza, soya y canola $(2,3)$.

Los LC-PUFAs son componentes fundamentales de los fosfolípidos presentes en todos nuestros tejidos y participan activamente en la regulación funcional de las membranas celulares y sub-celulares. Además, estos ácidos grasos esenciales participan en el control de fenómenos biológicos como el crecimiento celular, el metabolismo intracelular, la comunicación intercelular y la expresión de diversos genes que hoy son reconocidos como relevantes para la fisiopatología de enfermedades metabólicas y cardiovasculares (3). Después del tejido graso, el sistema nervioso es el principal sitio de depósito de los LC-PUFAs, y se ha demostrado que el déficit de LC-PUFAs se asocia a alteraciones en la formación y desarrollo de la corteza cerebral y la vía visual tanto en modelos animales como en humanos $(2,4)$.

Múltiples estudios han sugerido efectos benéficos de la suplementación con alimentos ricos en $\mathrm{DHA}$, reduciendo el riesgo y/o facilitando el manejo de patologías cardiovasculares, metabólicas, degenerativas y psiquiátricas (5-8); no obstante, no existe claridad en cuanto al momento ideal para apoyar la ingesta de este ácido graso esencial en la vida de un individuo. Se sabe que la acumulación de DHA en el organismo humano se produce primordialmente durante la segunda mitad del período de desarrollo fetal y los primeros meses de vida $(9,10)$, por este motivo se ha postulado que un apropiado traspaso de estos ácidos grasos desde la madre hacia el feto o recién nacido (lactancia materna), es de vital importancia para el desarrollo neurológico y visual. Adicionalmente, y siguiendo el planteamiento hipotético del origen temprano de las enfermedades crónicas (11-14), una adecuada disponibilidad de LC-PUFAs desde el período gestacional podría contribuir a la prevención del desarrollo de patologías cardiovasculares, metabólicas, degenerativas y psiquiátricas a largo plazo (15).

Con estos antecedentes, diversos estudios han evaluado el potencial beneficio de la suplementación dietaria con LC-PUFAs durante el embarazo sobre el resultado perinatal. En trabajos iniciales, principalmente observacionales, se ha sugerido que la alimentación rica en LC-PUFAs reduce la incidencia de pre-eclampsia, restricción de crecimiento intrauterino y parto prematuro (16-18).

Adicionalmente, se ha demostrado que la reducción en los depósitos maternos de DHA podría asociarse al desarrollo de síntomas depresivos en el período puerperal (19-22). Durante la gestación se ha evidenciado una disminución continua de los niveles maternos de DHA, especialmente en la segunda mitad del embarazo y la lactancia $(23,24)$. Esta reducción se explicaría por una activa extracción fetal y neonatal. Considerando estos antecedentes, la suplementación con ácidos grasos omega-3 y en especial con DHA ha sido propuesta como estrategia para la prevención y el manejo de la depresión post-parto.

El objetivo de este trabajo fue revisar la evidencia actualmente disponible, en investigaciones clínicas controladas y aleatorizadas, acerca de los beneficios de la suplementación prenatal con DHA sobre la madre y el recién nacido.

\section{MÉTODOS}

Criterios de inclusión. En este trabajo se consideró el análisis de investigaciones clínicas controladas y aleatorizadas (ICCA), que compararan la suplementación de DHA con placebo en embarazadas. Sólo se incluyó estudios realizados en pacientes con embarazos únicos de bajo riesgo, sin patologías del embarazo y con una adecuada caracterización de la población estudiada.

Se excluyó estudios donde se usaran los precursores directos del DHA, los ácidos linoleico y 
alfa-linolénico. Se consideró estudios con medición de resultados clínicos y bioquímicos. Los resultados clínicos incluidos fueron: tolerancia al tratamiento, edad gestacional al parto, incidencia de hipertensión gestacional y pre-eclampsia, antropometría neonatal y postnatal, síntomas depresivos maternos pre y post-parto, desarrollo neurológico general, desarrollo visual, nivel intelectual infantil y síntomas alérgicos en los primeros años de vida. Dentro de los resultados bioquímicos reportados, se incluyó aquellos trabajos referidos a los niveles de PUFAs en el plasma materno o neonatal, en células sanguíneas maternas y neonatales, así como en la leche materna. Por último, algunos trabajos recientes reportan resultados bioquímicos relativos al desarrollo del sistema inmune neonatal e infantil. Estrategia de búsqueda. La estrategia de búsqueda incluyó el uso de filtros específicos para localizar estudios clínicos aleatorizados y controlados en humanos. Los términos de búsqueda utilizados fueron: (docosahexaenoic acid or DHA or omega-3 or fish oil) and (pregnancy or maternal). El resultado de la búsqueda en MEDLINE (desde 1966 hasta enero 2012) fue inicialmente analizado en base al título y el resumen, para seleccionar aquellos que podrían ajustarse a los objetivos de la revisión. Estos trabajos fueron revisados en texto completo para evaluar su inclusión final en la revisión. La búsqueda fue complementada con el análisis de las referencias de cada trabajo incluido y con el análisis de revisiones previas disponibles. No hubo restricción de idioma en la búsqueda. No se incluyó cartas a editor, resúmenes de congresos o comunicaciones personales.

Análisis de calidad de los estudios. Se incluyó estudios de adecuada calidad metodológica, evaluada en cuanto al reporte del método de aleatorización y asignación de tratamiento, el riesgo de sesgos experimentales, el ocultamiento de información o ciego, el seguimiento efectivo en cada estudio y el análisis por intención de tratar.

\section{RESULTADOS}

Características generales de los estudios incluidos. El resultado de la búsqueda originó inicialmente 133 registros. De esta lista, se seleccionó 36 publicaciones que por el análisis del título y/o el resumen eran potencialmente relevantes para la revisión. Luego del análisis en texto completo, se incluyó finalmente 24 trabajos, correspondientes a resultados provenientes de estudios clínicos aleatorizados, controlados con placebo y doble-ciego. La lista de los trabajos de esta revisión incluyó 15 estudios que reportaron resultados clínicos (Tabla l) y 9 con resultados de laboratorio o bioquímicos (Tabla II). Las tablas respectivas describen las características generales de cada estudio.

Dosis y períodos de exposición evaluados. Se observó una amplia variabilidad en la dosis de DHA administrado como suplemento diario a las embarazadas (rango: $200-2.200 \mathrm{mg}$ ), siendo lo más común (38\% de los trabajos incluidos) la utilización de una dosis baja de aproximadamente $200 \mathrm{mg}$ de este ácido graso. Observamos en los trabajos más recientes una tendencia al uso de dosis mayores. La forma de administración también varió entre los distintos estudios, ya sea por el origen de los ácidos grasos (aceite de pescado, aceite de hígado de bacalao, aceite de algas), por la formulación (cápsulas, leche) o por la adición de otros suplementos en baja concentración (EPA, alfa-tocoferol, prebióticos como los fructo-oligosacáridos).

El período de exposición al suplemento se concentró en la segunda mitad del embarazo, con un promedio de inicio a las 20 semanas (rango: 12-30 semanas). Se observó una mayor variabilidad en el momento del término de la suplementación $(54 \%$ al momento del parto, $42 \%$ entre los 3 y 4 meses postparto, y sólo un trabajo administró el suplemento hasta los 6 meses post-parto). Este último trabajo correspondió a un estudio diseñado para evaluar la tolerancia y adhesión a la administración de los suplementos con ácidos grasos omega-3 (25). Todos los trabajos consideraron mujeres que planificaron dar lactancia materna.

Tolerancia materna. La tolerancia y la adherencia al protocolo de suplementación fueron evaluadas en forma secundaria en todos los trabajos revisados y en forma de objetivo principal en uno de ellos (25). Por esta razón, este último trabajo incluyó el rango más amplio de observación entre las 12 semanas de gestación y los 2 años post-parto. En este trabajo, un $19 \%$ de las pacientes que recibieron el suplemento con DHA versus un $25 \%$ de aquellas que recibieron el placebo, reportaron algún efecto secundarios. Estos efectos incluyeron fundamentalmente naúseas y vómitos, imposibilidad de tragar las cápsulas y la sensación de ineficacia del producto asignado. Sin embargo, tanto en este estudio específico como en el resto de los trabajos revisados, el porcentaje de pérdidas en el seguimiento o protocolo de estudio debido a mala tolerancia fue reducido en los distintos trabajos. De hecho, tomando los datos disponibles en la mayoría de las investigaciones incluidas, la declaración de mala tolerancia como causa de deserción del estudio fue en promedio de $3,4 \%$ versus $3,25 \%$ en el grupo de embarazadas que recibieron suplementación con DHA y placebo, respectivamente. 
Tabla I

ESTUDIOS CON MEDICIÓN DE RESULTADOS CLÍNICOS

\begin{tabular}{|c|c|c|c|}
\hline Autor (año) & $\begin{array}{l}\mathrm{DHA} \\
(\mathrm{mg})\end{array}$ & $\mathrm{n}$ & Medida de resultado \\
\hline Makrides (2010) & 800 & 2399 & $\begin{array}{l}\text { Síntomas depresivos post-parto y desarrollo } \\
\text { neurológico infantil }\end{array}$ \\
\hline Ramakrishnan (2010) & 400 & 1094 & $\begin{array}{l}\text { Edad gestacional al parto y antropometría } \\
\text { neonatal }\end{array}$ \\
\hline Stein (2011) & 400 & 739 & Crecimiento infantil a los 18 meses de vida \\
\hline Helland (2003) & 1183 & 341 & Nivel intelectual infantil a los 4 años \\
\hline Helland (2008) & 1183 & 341 & Nivel intelectual infantil a los 7 años \\
\hline Escolano-Margarit (2011) & 500 & 315 & Desarrollo neurológico infantil a los 4 y 5,5 años \\
\hline Rytter (2011) & 920 & 243 & IMC y perímetro abdominal a los 19 años \\
\hline Smithers (2011) & 800 & 185 & Maduración vía visual infantil \\
\hline Furuhjelm (2011) & 1100 & 145 & Síntomas de alergia a los 18 meses de vida \\
\hline Bergmann (2007) & 200 & 144 & Peso e IMC a los 21 meses de vida \\
\hline Doornbos (2008) & 220 & 119 & Síntomas depresivos maternos \\
\hline van Goor (2011) & 220 & 114 & $\begin{array}{l}\text { Desarrollo neurológico infantil a los } 18 \text { meses } \\
\text { de vida }\end{array}$ \\
\hline Freeman (2007) & 720 & 59 & Tolerancia al tratamiento \\
\hline Malcolm (2003a) & 200 & 56 & $\begin{array}{l}\text { Electroretinograma (función receptores visuales } \\
\text { de la retina) }\end{array}$ \\
\hline Malcolm (2003b) & 200 & 55 & Maduración vía visual infantil \\
\hline
\end{tabular}

Tabla II

ESTUDIOS CON MEDICIÓN DE RESULTADOS BIOQUÍMICOS

\begin{tabular}{|c|c|c|c|}
\hline Autor (año) & $\begin{array}{l}\mathrm{DHA} \\
(\mathrm{mg})\end{array}$ & $\mathrm{n}$ & Medida de resultado \\
\hline Stein(2011) & 400 & 739 & $\begin{array}{l}\text { Contenido DHA plasmático materno y neonatal, y } \\
\text { leche materna }\end{array}$ \\
\hline Helland (2006) & 1183 & 341 & $\begin{array}{l}\text { Contenido DHA plasmático materno y neonatal, y } \\
\text { leche materna }\end{array}$ \\
\hline Furuhjelm (2011) & 1100 & 145 & Respuesta inmune infantil \\
\hline Bergmann (2008) & 200 & 144 & DHA, EPA y AA en eritrocitos y leche materna \\
\hline Montgomery (2003) & 200 & 100 & $\begin{array}{l}\text { Contenido DHA plasmático, eritrocitos maternos y } \\
\text { neonatales, y leche materna }\end{array}$ \\
\hline Dunstan (2004) & 2200 & 83 & $\begin{array}{l}\text { DHA y EPA en eritrocitos maternos y neonatales. } \\
\text { Secundario }\end{array}$ \\
\hline van Goor (2009) & 220 & 69 & DHA y AA en leche materna \\
\hline Granot (2011) & 400 & 60 & Respuesta inmune en lactantes \\
\hline Sanjurjo (2004) & 200 & 16 & Contenido DHA plasmático materno y neonatal \\
\hline
\end{tabular}

DHA: ácido docosahexaenoico. EPA: ácido eicosapentaenoico. AA: ácido araquidónico. 
Efecto sobre los niveles de DHA. En general todos los trabajos que analizaron el efecto de la suplementación con DHA durante el embarazo y la lactancia, muestran que los niveles de este ácido graso en la madre se incrementan (26-32). El contenido de DHA en el plasma o en células sanguíneas maternas fue un 14 a $66 \%$ mayor en las mujeres que recibieron la suplementación, comparadas con aquellas que recibieron placebo. Este aumento fue mayor en función de la dosis de DHA suplementada.

Por otro lado, resulta interesante constatar que el contenido de DHA en la sangre de los niños o en la leche materna se vio aumentado en aquellos trabajos que usaron una dosis de DHA mayor $(27,28)$, o bien, que mantuvieron la suplementación durante el período de lactancia $(26,31)$. En los dos primeros estudios, la suplementación con DHA a dosis altas $(1,18 \mathrm{~g}$ o $2,2 \mathrm{~g}$ diarios) se asoció a un incremento de $48 \%$ en el contenido de DHA en la leche materna (28) y un aumento de $39 \%$ en el contenido de DHA en glóbulos rojos de los recién nacidos (27), en comparación a los grupos controles respectivos. En el caso de los trabajos que mantuvieron la suplementación con DHA durante la lactancia, se evidenció un aumento significativo de los niveles de este ácido graso en la leche materna, que fue de 42 a $100 \%$ mayor en las mujeres que recibieron la suplementación, comparadas con aquellas que recibieron el placebo $(26,31)$.

Efecto sobre síntomas depresivos maternos. Dos de los estudios más recientemente publicados evaluaron el potencial efecto de la suplementación con DHA sobre la depresión materna $(33,34)$. Makrides y cols (33), mostraron un $10,43 \%$ de pacientes con criterios diagnósticos para depresión post-parto según la Escala de Depresión Postnatal de Edimburgo (EPDS) durante los 6 primeros meses post-parto. A su vez, Doornbos y cols (34), evidenciaron un $6-7 \%$ de depresión y un $27 \%$ de distimia post-parto (postartum blues) en el grupo total de mujeres estudiadas con el mismo instrumento diagnóstico. Sin embargo, ambos trabajos mostraron que la suplementación materna con DHA no modifica la proporción de mujeres que presentaron síntomas depresivos tanto pre-natales como postnatales. La diferencia observada por Makrides y cols (33), en el diagnóstico de depresión post-parto para el grupo suplementado con DHA en comparación al grupo control, no fue estadísticamente significativa $(9,67 \%$ vs $11,19 \%$, respectivamente; RR 0,85; IC95\% 0,70-1,02; $p=0,09$ ); tampoco se observaron diferencias entre la proporción de pacientes con un nuevo diagnóstico de depresión ni en los requerimientos de tratamiento por patología psiquiátrica.

Efecto sobre el desarrollo cognitivo y visual infantil. El trabajo de Makrides y cols (33), antes mencionado, corresponde al estudio con mayor número de pacientes e incluye además la evaluación del desarrollo neurológico infantil, mediante la escala de evaluación del desarrollo infantil de Bayley. Los distintos aspectos del desempeño cognitivo y lenguaje de los niños a los 18 meses de vida no difirieron significativamente entre los grupos comparados, a excepción de la observación de un menor porcentaje de niños con retraso en el desarrollo cognitivo (puntuación < 85 en el ítem cognitivo de la escala Bayley) entre los hijos de madres que recibieron DHA (RR 0,4; IC95\% 0,22-0,78). Un estudio más reciente publicado por van Goor y cols (35), muestran que la suplementación materna (prenatal y durante los primeros meses de lactancia) con DHA a bajas dosis en combinación con ácido araquidónico no modifica el desempeño neurológico infantil a los 18 meses de vida.

Tres de los estudios incluidos en la revisión corresponden a una misma cohorte de pacientes que fueron aleatorizadas para recibir suplementación con una dosis alta de DHA (1,183 g diarios) o placebo, con el objetivo de evaluar el desarrollo cognitivo de los niños a largo plazo $(28,36,37)$. Así, un primer reporte da cuenta de un efecto benéfico de la suplementación sobre algunos aspectos de la capacidad de procesamiento mental a los 4 años de vida (36). En este reporte, se observó una mayor puntuación en la escala compuesta de procesamiento mental $\mathrm{K}-\mathrm{ABC}$ en el grupo de niños nacidos de mujeres que recibieron $\mathrm{DHA}$, en relación a aquellos provenientes del grupo control $(106,4 \pm 7,4$ versus 102,3 $\pm 11,3$; promedio \pm desviación estándar; $p=0,49$ ) (36). Estos datos se asociaron a una comprobación de un incremento significativo del contenido de DHA tanto en la madre como en la leche materna y en el niño (28). Sin embargo, el seguimiento de estos niños mostró que la suplementación con DHA no se asoció a una diferencia significativa en el nivel intelectual medido con la misma escala K-ABC a los 7 años (37). No obstante, este estudio mostró una correlación positiva entre el ítem específico de procesamiento secuencial de información a los 7 años y los niveles de DHA en el plasma materno a las 35 semanas de gestación $(r=0,196 ; p=0,021)$ (37). De la misma forma, un estudio reciente mostró que la suplementación materna con 500 mg diarios de DHA en combinación con $150 \mathrm{mg}$ de ácido araquidónico no modificó los índices de desarrollo neurológico infantil a los 4-5 años de vida (38). Este resultado negativo fue evidente a pesar de observarse una asociación positiva entre los mejores ín- 
dices de desarrollo medidos a los 5,5 años y los niveles de DHA en la sangre del cordón umbilical en los niños (38).

Adicionalmente, dos estudios provenientes del mismo grupo de trabajo evaluaron algunos aspectos del desarrollo de la vía visual infantil. Pacientes embarazadas fueron aleatorizadas a recibir DHA en bajas dosis (200 mg diarios) desde las 15 semanas de edad gestacional hasta el parto $(39,40)$. Posteriormente, los niños fueron evaluados con dos índices que estiman el grado de maduración de la capacidad de procesamiento de imágenes por el cerebro (39) y el funcionamiento de los fotoreceptores en la retina (40). Estos estudios no mostraron diferencias significativas entre los grupos comparados para ninguno de estos índices. Continuando en esta misma línea, Smithers y cols (41), publicaron recientemente que la suplementación materna con DHA en forma de cápsulas de aceite de pescado (800 mg diarios durante la segunda mitad del embarazo) no cambia las características de la respuesta visual infantil (potenciales eléctricos cerebrales evocados por estímulos visuales) a los 4 meses de vida; destaca en este estudio que la función visual evaluada de la forma descrita se aprecia significativamente alterada en los niños cuyas madres reportaron consumo de tabaco durante la gestación.

Efecto sobre la duración del embarazo, peso al nacer y crecimiento post-natal. El estudio de Makrides y cols (33), reporta una reducción significativa en la proporción de parto prematuro antes de las 34 semanas en el grupo de mujeres que recibieron suplementación con DHA, comparadas con el grupo control $(1,09 \%$ vs $2,25 \%$, respectivamente; RR 0,49 ; IC95\% 0,25-0,94; $p=0,03$ ). Adicionalmente, este estudio mostró un menor porcentaje de ingreso a UCI neonatal en el grupo suplementado con DHA, en relación al grupo control $(1,75 \%$ vs $3,08 \%$, respectivamente; RR 0,57; IC95\% 0,34-0,97; p=0,04), sin cambios en la mortalidad perinatal. Es importante comentar que estos resultados son parte del análisis secundario de este estudio multi-céntrico, enfocado en la prevención de síntomas depresivos maternos y el desarrollo neurológico infantil (33).

Por otra parte, cuando se evaluó dirigidamente la posibilidad de un efecto protector de la suplementación con DHA sobre la proporción de parto prematuro, los resultados fueron negativos (42). Este último estudio no observó diferencias entre el grupo de embarazadas que recibió el suplemento de DHA respecto al grupo que recibió placebo en la edad gestacional al parto $(39,1 \pm 1,7$ vs $39,0 \pm 1,9$ semanas, respectivamente; promedio \pm desviación estándar; $p>0,05)$ ni en la proporción de nacimientos antes de las 37 semanas $(10,1 \%$ vs $8,3 \%$, res- pectivamente; RR 1,2; IC95\% 0,8-1,8; $p=0,33)(42)$.

En cuanto al peso de los recién nacidos, el análisis secundario de los dos últimos estudios publicados por Makrides y cols (33) y Ramakrishnan y cols (42) mostraron un promedio de peso al nacer significativamente mayor en mujeres que recibieron DHA. En el primer estudio, se reporta una menor proporción de recién nacidos con un peso menor a $2500 \mathrm{~g}$ en el grupo de mujeres que recibieron la suplementación con DHA, comparadas con el grupo control $(3,41 \%$ vs $5,27 \%$, respectivamente; RR 0,65; IC95\% 0,44-0,96; $p=0,03$ ) (33). No obstante, las diferencias de peso al nacer entre los grupos analizados por Makrides y cols (33) desaparecieron luego de ajustar por edad gestacional al parto y sexo neonatal. Por su parte, Ramakrishnan y cols (42), mostraron que sólo en el caso de pacientes primigestas que recibieron suplementación con DHA se reducía el riesgo de presentar restricción de crecimiento intrauterino (peso menor al percentil 10 para la edad gestacional), en comparación al grupo de pacientes primigestas que recibieron placebo $(7,1 \%$ vs $14 \%$, respectivamente; RR 0,5 ; IC95\% 0,3-1,0; $p=0,03$ ).

En un reporte más reciente, el grupo de Ramakrishnan y cols (43), mostró que el crecimiento infantil evaluado a los 18 meses de vida no se modifica significativamente en la cohorte de niños cuyas madres recibieron DHA. Al igual que lo observado en el caso del crecimiento fetal, sólo el subgrupo de hijos de mujeres primigestas que recibió DHA mostró una estatura mayor a los 18 meses, comparado con los hijos de mujeres primigestas que recibió placebo (43). En este mismo sentido, un estudio previo evaluó el efecto de la suplementación materna con DHA sobre el estado nutricional posterior de los niños, evaluados a los 21 meses de vida. Aunque este trabajo reporta que el promedio de índice de masa corporal (IMC) en el grupo de niños cuyas madres recibieron suplementación con DHA tiende a ser menor en comparación al grupo control a esta edad (44), la diferencia entre ambos grupos no fue significativa (IMC 14,7 $\pm 0,36$ vs $15,46 \pm 0,32$, respectivamente; $p=0,12$ ). Por último, recientemente se han publicado los primeros resultados del seguimiento a mediano plazo de los hijos de mujeres que participaron en un estudio de suplementación con aceite de pescado enriquecido en DHA y EPA durante el embarazo (45). En este análisis se evaluó el IMC, el perímetro de cintura y algunos marcadores bioquímicos de adiposidad en los grupos de jóvenes cuyas madres recibieron el suplemento o placebo. En concordancia con los estudios anteriores, en este seguimiento no se observaron diferencias significativas entre los grupos 
comparados (45).

Efecto sobre síntomas de alergia y desarrollo del sistema inmune. Una serie de estudios recientes ha evaluado el potencial rol regulador de la suplementación prenatal con DHA sobre el sistema inmune en desarrollo. Uno de estos trabajos reporta los resultados de la exposición con DHA a dosis altas $(1,1 \mathrm{~g}$ al día) en combinación con EPA y por un período prolongado (25 semanas de gestación hasta 3,5 meses post-parto) sobre la incidencia de síntomas de enfermedades alérgicas asociadas a niveles elevados de Inmunoglobulina E (46). Aunque los niveles plasmáticos de DHA elevados en las mujeres y sus hijos se asociaron a una menor proporción de síntomas alérgicos, el análisis por intención de tratar no mostró diferencias en la incidencia de las patologías alérgicas evaluadas a los 2 años de vida entre los grupos comparados (46). Un segundo reporte de este mismo grupo de estudio evidencia que la suplementación materna con altas dosis de DHA produjo un cambio en la capacidad de respuesta inmune infantil. Este cambio consistió en un aumento de la respuesta bioquímica dependiente de la activación de linfocitos T-helper 1 (Th1) en desmedro de la respuesta Th2 (47). En este mismo ámbito, un tercer estudio mostró que la suplementación materna con DHA (400 mg diarios desde las 12 semanas hasta 4 meses post-parto) se asoció a una atenuación de la respuesta inmune infantil (48). En este trabajo, el grupo de niños nacidos de mujeres que recibieron el suplemento evidenció una disminución en la producción de citoquinas pro-inflamatorias en linfocitos CD4 y CD8, con una mayor proporción de células CD4 inactivas (48).

\section{DISCUSIÓN}

Este trabajo analizó la mejor evidencia disponible acerca de los potenciales efectos benéficos de la suplementación materna con el ácido graso esencial DHA sobre la salud materno-infantil. Por un lado, la gran mayoría de las 24 ICCA incluidas en esta revisión confirmaron que la suplementación con DHA aumenta el contenido de DHA en el plasma y la leche materna, favoreciendo la disponibilidad de este ácido graso para la nutrición fetal y neonatal. Además, en aquellos estudios donde se usaron dosis mayores de DHA, los niveles de DHA en el plasma o los glóbulos rojos neonatales también fueron aumentados. Sin embargo, la información derivada de los estudios que evaluaron parámetros clínicos nos parece aún insuficiente para apoyar la suplementación prenatal con DHA. En general, el conjunto de resultados mostró que la suplementación nutricional con este ácido graso no se asoció a beneficios clínicamente significativos en cuanto a prevención de depresión materna, desarrollo neurológico y visual infantil, duración del embarazo, peso al nacer, crecimiento post-natal o patologías alérgicas infantiles.

Los estudios clínicos incluidos en este análisis muestran que la suplementación materna con DHA se asocia a un mayor contenido de LC-PUFAs en el compartimento materno. Dependiendo de la dosis recibida o el tiempo de suplementación, se observó una mayor acumulación en la leche materna y en el plasma de los niños. En los trabajos revisados se apreció que el mayor aporte de LC-PUFAs en la dieta de la mujer embarazada contribuyó a evitar la reducción fisiológica de los niveles plasmáticos de estos ácidos grasos durante la gestación. Tomando en cuenta que en algunas poblaciones el consumo de estos ácidos grasos en la dieta podría encontrarse disminuido, el efecto de la suplementación con LC-PUFAs evitaría las posibles consecuencias deletéreas derivadas de un déficit perinatal excesivo de DHA u otros ácidos grasos esenciales. No obstante, pese a que los estudios reportan aumentos significativos en el contenido de DHA en la leche materna y el neonato en distintas etapas, la información referida a la potencial relevancia clínica de estos cambios aún es discordante.

Los estudios que evaluaron los efectos de la suplementación materna con DHA sobre resultados clínicos como la depresión materna o el desarrollo neurológico, visual y cognitivo de los niños son contradictorios. En general, la suplementación con DHA durante el embarazo no se asoció a una reducción en la presentación de síntomas depresivos maternos ni a una mejoría global en la capacidad de procesamiento cognitivo y visual en los niños estudiados. Estos hallazgos podrían explicarse por diversas razones. En primer lugar, es posible que efectivamente no exista un beneficio derivado de la suplementación prenatal con DHA para prevenir síntomas depresivos en la madre o para mejorar el desarrollo neurológico de los niños. Sin embargo, debido a que algunos elementos específicos de la evaluación cognitiva en niños expuestos a DHA mostraron mejorías significativas en la capacidad de procesamiento mental a los 4 y 7 años $(36,37)$ o en el riesgo de retraso neurológico a los 18 meses (33), es posible que sólo ciertos aspectos del desarrollo neurológico infantil puedan ser favorecidos por la suplementación materna con DHA. Dado que el contenido de ácidos grasos esenciales puede variar entre distintos sectores del sistema nervioso humano, es probable que cambios nutricionales que involucren una mayor biodisponibilidad de DHA 
afecte sólo a algunos aspectos del desarrollo neurológico infantil (35).

Los estudios referidos en el análisis de resultados psiquiátricos maternos o neurológicos infantiles, muestran características nutricionales basales comparables entre las poblaciones evaluadas. Sin embargo, el reducido número de individuos en los grupos comparados hace posible que los resultados se vean influidos por variables adicionales como el nivel socio-económico o las características ambientales que condicionen un mayor o menor riesgo de depresión o alteraciones del desarrollo neurológico infantil. Estas posibilidades no fueron evaluadas en los estudios analizados. Adicionalmente, la dosis de DHA contenida en los diferentes suplementos evaluados, así como los períodos de suplementación y/o evaluación podrían no ser los adecuados. Por un lado, la baja dosis de DHA (200 mg diarios) suplementada en los estudios de Malcolm y cols $(39,40)$, constituye uno de los eventuales factores que expliquen la ausencia de beneficio en el desarrollo visual infantil. Sin embargo, esta posibilidad fue descartada en parte por el estudio de Smithers y cols (41), en donde aún con una dosis de $800 \mathrm{mg}$ diarios de DHA no se observaron efectos a nivel de la respuesta visual en los lactantes de 4 meses. En todo caso, no es posible descartar que potenciales beneficios de la suplementación materna con DHA sobre resultados neurológicos infantiles a largo plazo aún no hayan sido reportados, solamente debido al corto tiempo de seguimiento actual.

En esta revisión sólo se incluyó trabajos que consideraban embarazadas de bajo riesgo sin complicaciones, donde la suplementación con DHA fue usada para prevenir el desarrollo de patologías o mejorar los resultados perinatales. De esta forma, los eventuales efectos adicionales de los LCPUFAs en embarazadas de riesgo o con alguna condición patológica quedaron fuera del análisis. En este contexto, destaca el beneficio del uso de LC-PUFAs en mujeres con trastorno depresivo mayor diagnosticado durante la gestación, reportado por algunos trabajos no incluidos en la revisión. En mujeres embarazadas con depresión, la suplementación nutricional con DHA disminuye la sintomatología asociada luego de 6 y 8 semanas de tratamiento (49). Estos resultados se valoran aún más a la luz de otros estudios que muestran que el uso de DHA solamente durante el período post-parto no modifica los síntomas depresivos ni la capacidad de procesamiento de información de las mujeres (23). Así, el uso de DHA podría considerarse más útil en el tratamiento de la depresión materna que en su prevención.
En cuanto al resultado perinatal, existe controversia entre los efectos de la suplementación con DHA sobre el riesgo de parto prematuro o la restricción de crecimiento intrauterino. Estudios previos observacionales mostraron un aumento significativo en la duración del embarazo en mujeres que recibieron una alimentación enriquecida en DHA (16), concordando con la reducción en la proporción de parto prematuro menor a las 34 semanas mostrada por una de las ICCA incluidas en nuestra revisión (33). Sin embargo, estos hallazgos no fueron reproducidos en un trabajo diseñado específicamente para evaluar el beneficio de la suplementación con DHA y el parto prematuro (42). Como hemos discutido para otros resultados evaluados, estas inconsistencias podrían deberse a diferencias nutricionales entre las poblaciones estudiadas, diferencias raciales, paridad materna, entre otras. Especial énfasis debe hacerse en las posibles diferencias iniciales en los niveles de DHA y otros ácidos grasos esenciales, previo a la suplementación de las distintas poblaciones. Adicionalmente, destaca que en el estudio de Makrides y cols (33), la dosis de DHA fue el doble de la dosis suplementada a las embarazadas en el estudio de Ramakrishnan y cols (42) (800 mg vs 400 mg diarios). De este modo, la poca contundencia del primer estudio (disminución del riesgo de parto prematuro antes de las 34 semanas, pero sin diferencias generales en la edad gestacional al parto) y la ausencia de resultados positivos en el segundo estudio, podrían deberse a una dosis aún reducida de DHA.

Un meta-análisis que agrupó estudios sobre el efecto de la suplementación con LC-PUFAs en el resultado perinatal mostró un beneficio global sobre la duración del embarazo (50). Sin embargo, la escasa magnitud de las diferencias reportadas (un promedio de 1,6 días más de duración del embarazo y sólo un aumento significativo de la circunferencia craneana en el grupo que recibió LC-PUFAs) indica que aún no existe evidencia suficiente para sustentar un efecto clínicamente significativo sobre la prolongación del embarazo o el crecimiento fetal. No obstante, podemos destacar datos adicionales derivados del análisis secundario realizado en el estudio multi-céntrico de Makrides y cols (33); en este estudio, junto con mostrar una reducción en la incidencia de parto prematuro antes de las 34 semanas, se informa un menor ingreso a $\mathrm{UCI}$ neonatal entre los hijos de mujeres que recibieron la suplementación prenatal con DHA. Aun cuando el conjunto de los estudios no demuestra una prolongación significativa de los embarazos en respuesta a la suplementación con DHA, una reducción de 
los nacimientos antes de las 34 semanas podría constituir un efecto específico potencialmente relevante de esta intervención. Los trabajos publicados no reportan los datos suficientes para analizar esta posibilidad en particular. Considerando los antecedentes descritos, es posible que nuevos diseños de ICCA puedan evaluar dirigidamente la utilidad de la suplementación con dosis altas de DHA sobre el riesgo de parto prematuro.

Los estudios que evaluaron la capacidad de la suplementación con DHA prenatal para prevenir el parto prematuro, muestran además datos acerca del crecimiento fetal en respuesta a esta intervención nutricional. En primer lugar, Makrides y cols (33) muestran una menor proporción de recién nacidos con un peso bajo $2500 \mathrm{~g}$ en el grupo de mujeres suplementadas con DHA. Sin embargo, estos autores reconocen que la diferencia en los promedios de peso al nacer de ambos grupos comparados, desaparece luego de ajustar por edad gestacional. Con esto, se sugiere que el efecto de la suplementación materna con DHA sobre el crecimiento fetal se explica por la menor proporción de partos prematuros antes de las 34 semanas observada en su análisis. Cualquiera que sea el eventual efecto primario de la suplementación materna con DHA en este contexto, si es posible demostrarlo en nuevos estudios, constituiría una potente razón para utilizar dicha intervención en el embarazo. En segundo lugar, dentro del trabajo publicado por Ramakrishnan y cols (42) es posible detectar un efecto selectivo de la suplementación con DHA, dependiendo de las características de las pacientes. El análisis de subgrupos en ese estudio mostró que el riesgo de un bajo peso para la edad gestacional en las embarazadas primigestas que recibieron suplemento con DHA era menor que en las primigestas que recibieron el placebo. Al igual que la mayor estatura alcanzada por hijos de mujeres primigestas que recibieron suplementación con DHA (43), la diferencia en el riesgo de bajo peso fetal no se observó en las pacientes multíparas o en el grupo total de embarazadas $(42,43)$.

Un eficacia selectiva del DHA para aumentar el peso del recién nacido podría revelar escenarios de acción más focalizados, en los que la suplementación sea favorable. De igual relevancia podría ser la información reportada por Bergmann y cols (44), quienes mostraron que la suplementación materna con DHA se asoció a una progresión potencialmente protectora del IMC hasta los 21 meses de vida en hijos de las mujeres que recibieron la suplementación. Sin embargo, la propuesta de un potencial rol de la suplementación materna con DHA en la prevención de obesidad fue descartada al menos a mediano plazo. Esto debido a la ausencia de diferencias en los índices antropométricos y bioquímicos en un reporte del seguimiento nutricional en jóvenes de 19 años cuyas madres recibieron aceite de pescado enriquecido en DHA (45).

La estrecha relación existente entre los ácidos grasos y la generación de mediadores inflamatorios ha contribuido a la propuesta de un potencial rol de los niveles circulantes de DHA en el desarrollo del sistema inmune infantil (48). Efectivamente, los estudios analizados muestran que es factible una cierta modulación de la capacidad de respuesta inmune infantil, al menos a nivel bioquímico $(47,48)$. Sin embargo, aunque tales modificaciones deberían asociarse a un menor riesgo de enfermedades alérgicas en los niños, la incidencia de los síntomas clínicos de estas patologías a los 2 años de vida no se modifica en respuesta a la suplementación materna con DHA (46).

En resumen, la evidencia disponible sobre la utilidad de la suplementación con LC-PUFAs y especialmente con DHA durante el embarazo, presenta limitaciones derivadas principalmente de la gran variabilidad en relación a dosis, tiempo de exposición y resultados materno-perinatales evaluados. De este modo, aún no es posible acumular un número razonable de pacientes que permita realizar un metanálisis homogéneo para extraer conclusiones válidas desde el punto de vista clínico. Los trabajos analizados permiten sostener que existen potenciales efectos benéficos de la suplementación materna con DHA que requieren estudios adicionales bien diseñados. En este sentido, los estudios revisados plantean la necesidad de evaluar con mayor detención los potenciales efectos sobre aspectos puntuales del desarrollo cognitivo infantil. De igual forma, es posible plantear la evaluación específica de un rol protector de la suplementación prenatal con DHA sobre el riesgo de parto prematuro menor a 34 semanas y la restricción de crecimiento fetal. Esto último sería especialmente factible en grupos de riesgo como por ejemplo pacientes primigestas con déficit nutricional.

Un aspecto importante a considerar en el análisis y el planteamiento de estos trabajos corresponde a la cantidad de DHA que deben aportar los suplementos nutricionales utilizados. Por un lado, se conocen estimaciones epidemiológicas de los niveles recomendados de ingesta diaria de DHA necesario para cubrir los requerimientos de un porcentaje cercano al $100 \%$ de la población que bordean los $800 \mathrm{mg}$ (51). Por otro lado, existe evidencia de la seguridad farmacológica de dosis elevadas de este ácido graso administrado durante la gestación $(25,36)$. Aunque esta revisión muestra que aún no 
es posible determinar la dosis óptima recomendada para obtener los mejores resultados perinatales, las discrepancias en los distintos resultados podría deberse a una baja dosis de DHA recibida por las mujeres en aquellos estudios sin resultados favorables. Considerando estos datos, parece razonable plantear que futuros estudios orientados a dilucidar eventuales efectos de la suplementación prenatal con DHA utilicen al menos $800 \mathrm{mg}$ diarios de este ácido graso. Adicionalmente, la evaluación de la ingesta habitual y los niveles basales de ácidos grasos esenciales disponibles en las diferentes poblaciones de embarazadas, podría contribuir a una mejor estimación de las dosis requeridas en futuros estudios.

Uno de los hallazgos más frecuentes en este tópico es una serie de asociaciones significativas evidenciadas entre los niveles circulantes o celulares de DHA tanto en la madre como en los niños expuestos o no a la suplementación materna, con los diversos resultados medidos. Sin embargo, los estudios clínicos no logran demostrar diferencias relevantes entre los grupos comparados. Este tipo de resultados hace necesario descartar eventuales factores adicionales que puedan explicar un efecto parcialmente benéfico de la suplementación prenatal con DHA sobre parámetros bioquímicos y no clínicos. Entre estos factores destacan las potenciales diferencias entre las poblaciones estudiadas (variables socio-económicas, ambientales, nutricionales, etc.), las diferentes dosis administradas y sus formulaciones, la interferencia de otros suplementos recibidos durante el estudio y los tiempos de exposición.

Una hipótesis interesante sostiene que los niveles de DHA en los diferentes compartimentos en donde se ha podido medir pueden corresponder a una valoración subyacente al estado nutricional o al nivel socio-económico o cultural de un individuo o población (35). De este modo, los niveles elevados de DHA podrían corresponder no sólo a un marcador de una dieta enriquecida en ácidos grasos esenciales, sino también a una aproximación a un estilo de vida saludable que incluya muchas otras variables (nutrientes, conductas, estímulos, etc.). Si esto es correcto, es esperable que una intervención nutricional tan específica no sea completamente beneficiosa desde el punto de vista clínico, aún cuando se observen correlaciones entre los niveles plasmáticos del nutriente aportado y el resultado buscado.

\section{CONCLUSIÓN}

Las diferencias de efectividad entre los distin- tos estudios hacen necesaria una estricta validación poblacional local. Un análisis que considere las diferencias étnicas, entre otros posibles factores que puedan confundir, es fundamental para la decisión de aceptación o desestimación definitiva de intervenciones que probablemente dependen de las características nutricionales y antropométricas de los individuos estudiados. La suplementación prenatal con DHA produce un aumento en los niveles maternos y neonatales de este ácido graso. En principio, es posible sostener que los estudios disponibles apoyan la necesidad de evaluar de manera dirigida el potencial efecto benéfico de la suplementación prenatal con DHA sobre la duración de la gestación, el peso al nacer y el desarrollo cognitivo neonatal e infantil, además de los eventuales beneficios de este ácido graso para la disminución de síntomas depresivos maternos. Tales evaluaciones deberán considerar el estado basal de ácidos grasos disponibles en la mujer embarazada y las dosis seguras y eficaces para cada población. En virtud de la información actual, creemos que aún no existe evidencia suficiente para sustentar la recomendación universal de suplementos nutricionales con DHA durante la gestación como medida preventiva perinatal. Sin embargo, recomendamos la realización de nuevos estudios clínicos controlados aleatorizados que permitan resolver la potencial utilidad del DHA en grupos de riesgo definidos por patologías que se beneficien de su uso o por una disponibilidad inadecuada de este ácido graso.

\section{REFERENCIAS}

1. Ratnayake WM, Galli C. Fat and fatty acid terminology, methods of analysis and fat digestion and metabolism: a background review paper. Ann Nutr Metab 2009;55:8-43.

2. Uauy $R$, Dangour $A D$. Fat and fatty acid requirements and recommendations for infants of 0-2 years and children of 2-18 years. Ann Nutr Metab 2009;55:76-96.

3. Jung UJ, Torrejon C, Tighe AP, Deckelbaum RJ. n-3 Fatty acids and cardiovascular disease: mechanisms underlying beneficial effects. Am J Clin Nutr 2008;87:2003S-2009S.

4. Innis SM. Essential fatty acid transfer and fetal development. Placenta 2005;26(Suppl A):S70-75.

5. Zevenbergen $\mathrm{H}$, de Bree $\mathrm{A}$, Zeelenberg $\mathrm{M}$, Laitinen $\mathrm{K}$, van Duijn G, Flöter E. With a high fat quality are essential for healthy diets. Ann Nutr Metab 2009;54(Suppl 1):15-24.

6. Ross BM, Seguin J, Sieswerda LE. Omega-3 fatty acids as treatments for mental illness: which disorder and which fatty acid? Lipids Health Dis 2007;6:21.

7. Nettleton JA, Katz R. n-3 long-chain polyunsaturated fatty acids in type 2 diabetes: a review. J Am Diet Assoc 2005;105:428-40.

8. Jicha GA, Markesbery WR. Omega-3 fatty acids: po- 
tential role in the management of early Alzheimer's disease. Clin Interv Aging 2010;5:45-61.

9. Martínez M, Mougan I. Fatty acid composition of human brain phospholipids during normal development. J Neurochem 1998;71:2528-33.

10. AI MD, van Houwelingen AC, Hornstra G. Long-chain polyunsaturated fatty acids, pregnancy, and pregnancy outcome. Am J Clin Nutr 2000;71:285S-91S.

11. Symonds ME, Sebert SP, Hyatt MA, Budge H. Nutritional programming of the metabolic syndrome. Nat Rev Endocrinol 2009;5:604-10.

12. McMillen IC, Robinson JS. Developmental origins of the metabolic syndrome: prediction, plasticity, and programming. Physiol Rev 2005;85:571-633.

13. Bouret SG. Early life origins of obesity: role of hypothalamic programming. J Pediatr Gastroenterol Nutr 2009;48(Suppl 1):S31-8.

14. Bruce KD, Hanson MA. The developmental origins, mechanisms, and implications of metabolic syndrome. J Nutr 2010;140:648-52.

15. Innis SM. Metabolic programming of long-term outcomes due to fatty acid nutrition in early life. Matern Child Nutr 2011;7(Suppl 2):112-23.

16. Olsen SF, Østerdal ML, Salvig JD, Kesmodel U, Henriksen TB, Hedegaard M, et al. Duration of pregnancy in relation to seafood intake during early and mid pregnancy: prospective cohort. Eur $\mathrm{J}$ Epidemiol 2006;21:749-58

17. Oken E, Ning Y, Rifas-Shiman SL, Rich-Edwards JW, Olsen SF, Gillman MW. Diet during pregnancy and risk of preeclampsia or gestational hypertension. Ann Epidemiol 2007;17:663-8.

18. Halldorsson TI, Meltzer HM, Thorsdottir I, Knudsen $\mathrm{V}$, Olsen SF. Is high consumption of fatty fish during pregnancy a risk factor for fetal growth retardation? A study of 44,824 Danish pregnant women. Am J Epidemiol 2007;166:687-96.

19. Suominen-Taipale AL, Partonen T, Turunen AW, Männistö S, Jula A, Verkasalo PK. Fish consumption and omega-3 polyunsaturated fatty acids in relation to depressive episodes: a cross-sectional analysis. PLoS One 2010;5:e10530.

20. Leung BM, Kaplan BJ. Perinatal depression: prevalence, risks, and the nutrition link--a review of the literature. J Am Diet Assoc 2009;109:1566-75.

21. Hibbeln JR. Seafood consumption, the DHA content of mothers' milk and prevalence rates of postpartum depression: a cross-national, ecological analysis. J Affect Disord 2002;69:15-29.

22. Freeman MP. Omega-3 fatty acids and perinatal depression: a review of the literature and recommendations for future research. Prostaglandins Leukot Essent Fatty Acids 2006;75:291-7.

23. Llorente AM, Jensen CL, Voigt RG, Fraley JK, Berretta MC, Heird WC. Effect of maternal docosahexaenoic acid supplementation on postpartum depression and information processing. Am J Obstet Gynecol 2003;188:1348-53.

24. Al MD, van Houwelingen AC, Kester AD, Hasaart TH, de Jong $A E$, Hornstra G. Maternal essential fatty acid patterns during normal pregnancy and their relations- hip to the neonatal essential fatty acid status. Br J Nutr 1995; 74:55-68.

25. Freeman MP, Sinha P. Tolerability of omega-3 fatty acid supplements in perinatal women. Prostaglandins Leukot Essent Fatty Acids 2007;77:203-8.

26. Bergmann RL, Haschke-Becher E, Klassen-Wigger $\mathrm{P}$, Bergmann KE, Richter R, Dudenhausen JW, et al. Supplementation with $200 \mathrm{mg} /$ day docosahexaenoic acid from mid-pregnancy through lactation improves the docosahexaenoic acid status of mothers with a habitually low fish intake and of their infants. Ann Nutr Metab 2008;52:157-66.

27. Dunstan JA, Mori TA, Barden A, Beilin LJ, Holt PG, Calder PC, et al. Effects of $n-3$ polyunsaturated fatty acid supplementation in pregnancy on maternal and fetal erythrocyte fatty acid composition. Eur J Clin Nutr 2004;58:429-37.

28. Helland IB, Saugstad OD, Saarem K, Van Houwelingen AC, Nylander G, Drevon CA. Supplementation of $\mathrm{n}-3$ fatty acids during pregnancy and lactation reduces maternal plasma lipid levels and provides DHA to the infants. J Matern Fetal Neonatal Med 2006;19:397406.

29. Montgomery C, Speake BK, Cameron A, Sattar N, Weaver LT. Maternal docosahexaenoic acid supplementation and fetal accretion. Br J Nutr 2003;90:13545.

30. Sanjurjo P, Ruiz-Sanz JI, Jimeno P, Aldámiz-Echevarría L, Aquino L, Matorras R, et al. Supplementation with docosahexaenoic acid in the last trimester of pregnancy: maternal-fetal biochemical findings. J Perinat Med 2004;32:132-6.

31. van Goor SA, Dijck-Brouwer DA, Hadders-Algra M, Doornbos B, Erwich JJ, Schaafsma A et al. Human milk arachidonic acid and docosahexaenoic acid contents increase following supplementation during pregnancy and lactation. Prostaglandins Leukot Essent Fatty Acids 2009;80:65-9.

32. Imhoff-Kunsch B, Stein AD, Villalpando S, Martorell R, Ramakrishnan U. Docosahexaenoic acid supplementation from mid-pregnancy to parturition influenced breast milk fatty acid concentrations at 1 month postpartum in mexican women. J Nutr 2011;141:321-6.

33. Makrides M, Gibson RA, McPhee AJ, Yelland L, Quinlivan J, Ryan P; DOMInO Investigative Team. Effect of DHA supplementation during pregnancy on maternal depression and neurodevelopment of young children: a randomized controlled trial. JAMA 2010;304:167583.

34. Doornbos B, van Goor SA, Dijck-Brouwer DA, Schaafsma A, Korf J, Muskiet FA. Supplementation of a low dose of DHA or DHA+AA does not prevent peripartum depressive symptoms in a small population based sample. Prog Neuropsychopharmacol Biol Psychiatry 2009;33:49-52.

35. van Goor SA, Dijck-Brouwer DA, Erwich JJ, Schaafsma A, Hadders-Algra M. The influence of supplemental docosahexaenoic and arachidonic acids during pregnancy and lactation on neurodevelopment at eighteen months. Prostaglandins Leukot Essent Fatty Acids 2011;84:139-46. 
36. Helland IB, Smith L, Saarem K, Saugstad OD, Drevon $\mathrm{CA}$. Maternal supplementation with very-longchain n-3 fatty acids during pregnancy and lactation augments children's IQ at 4 years of age. Pediatrics 2003;111:e39-44.

37. Helland IB, Smith L, Blomén B, Saarem K, Saugstad OD, Drevon CA. Effect of supplementing pregnant and lactating mothers with $n-3$ very-long-chain fatty acids on children's IQ and body mass index at 7 years of age. Pediatrics 2008;122:e472-9.

38. Escolano-Margarit MV, Ramos R, Beyer J, Csábi G, Parrilla-Roure M, Cruz $\mathrm{F}$ et al. Prenatal DHA status and neurological outcome in children at age 5.5 years are positively associated. J Nutr 2011;141:1216-1223.

39. Malcolm CA, McCulloch DL, Montgomery C, Shepherd A, Weaver LT. Maternal docosahexaenoic acid supplementation during pregnancy and visual evoked potential development in term infants: a double blind, prospective, randomised trial. Arch Dis Child Fetal Neonatal Ed 2003;88:F383-90.

40. Malcolm CA, Hamilton R, McCulloch DL, Montgomery C, Weaver LT. Scotopic electroretinogram in term infants born of mothers supplemented with docosahexaenoic acid during pregnancy. Invest Ophthalmol Vis Sci 2003; 44:3685-91.

41. Smithers LG, Gibson RA, Makrides M. Maternal supplementation with docosahexaenoic acid during pregnancy does not affect early visual development in the infant: a randomized controlled trial. Am J Clin Nutr 2011;93:1293-9.

42. Ramakrishnan $U$, Stein $A D$, Parra-Cabrera $S$, Wang $\mathrm{M}$, Imhoff-Kunsch B, Juárez-Márquez $\mathrm{S}$, et al. Effects of docosahexaenoic acid supplementation during pregnancy on gestational age and size at birth: randomized, double-blind, placebo-controlled trial in Mexico. Food Nutr Bull 2010;31:S108-16.

43. Stein AD, Wang M, Martorell R, Neufeld LM, FloresAyala R, Rivera JA, et al. Growth to age 18 months following prenatal supplementation with docosahexaenoic acid differs by maternal gravidity in mexico. J Nutr
2011;141:316-20.

44. Lucia Bergmann R, Bergmann KE, Haschke-Becher E, Richter R, Dudenhausen JW, Barclay D, et al. Does maternal docosahexaenoic acid supplementation during pregnancy and lactation lower BMI in late infancy? J Perinat Med 2007;35:295-300.

45. Rytter D, Bech BH, Christensen JH, Schmidt EB, Henriksen TB, Olsen SF. Intake of fish oil during pregnancy and adiposity in 19-y-old offspring: followup on a randomized controlled trial. Am J Clin Nutr 2011;94:701-8.

46. Furuhjelm $C$, Warstedt K, Fagerås M, Fälth-Magnusson K, Larsson J, Fredriksson M, et al. Allergic disease in infants up to 2 years of age in relation to plasma omega-3 fatty acids and maternal fish oil supplementation in pregnancy and lactation. Pediatr Allergy Immunol 2011;22:505-14.

47. Furuhjelm C, Jenmalm MC, Fälth-Magnusson K, Duchén $\mathrm{K}$. Th1 and Th2 chemokines, vaccine-induced immunity, and allergic disease in infants after maternal omega-3 fatty acid supplementation during pregnancy and lactation. Pediatr Res 2011;69:259-64.

48. Granot E, Jakobovich E, Rabinowitz R, Levy P, Schlesinger M. DHA supplementation during pregnancy and lactation affects infants' cellular but not humoral immune response. Mediators Inflamm 2011;2011:493925.

49. Su KP, Huang SY, Chiu TH, Huang KC, Huang CL, Chang $\mathrm{HC}$, et al. Omega-3 fatty acids for major depressive disorder during pregnancy: results from a randomized, double-blind, placebo-controlled trial. J Clin Psychiatry 2008;69:644-51.

50. Szajewska $H$, Horvath A, Koletzko B. Effect of $n-3$ long-chain polyunsaturated fatty acid supplementation of women with low-risk pregnancies on pregnancy outcomes and growth measures at birth: a metaanalysis of randomized controlled trials. Am J Clin Nutr 2006;83:1337-44.

51. Hibbeln JR, Davis JM. Considerations regarding neuropsychiatric nutritional requirements for intakes of omega-3 highly unsaturated fatty acids. Prostaglandins Leukot Essent Fatty Acids 2009;81:179-86. 